Title

\title{
Extra collagen overlay prolongs the differentiated phenotype in sandwich- cultured rat hepatocytes
}

Marlies Oorts ${ }^{1,{ }^{*}}$, Janneke Keemink $^{1,2,{ }^{*}}$, Neel Deferm ${ }^{1}$, Robin Adriaensen ${ }^{1,3}$, Lysiane Richert ${ }^{4,5}$, Patrick Augustijns ${ }^{1}$, Pieter Annaert ${ }^{1, * *}$

1: Drug Delivery and Disposition, KU Leuven Department of Pharmaceutical and Pharmacological Sciences, O\&N2, Herestraat 49 - bus 921, 3000 Leuven, Belgium

2: Uppsala University, Department of Pharmacy, Box 580, 75123 Uppsala, Sweden (present address)

3: Quality Assurance, GSK Consumer Healthcare Benelux, Site Apollo, Avenue Pascal 2-4-6, 1300 Wavre, Belgium. (present address)

4: KaLy-Cell, 20 A rue du Général Leclerc, 67115 Plobsheim, France

5: Université de Bourgogne Franche-Comté, EA 4267, Besançon, France

* These authors contributed equally to this work

** Corresponding author. Contact: +3216330303 or pieter.annaert@kuleuven.be 


\section{ABSTRACT}

Introduction: Sandwich-cultured rat hepatocytes (SCRH) have become an invaluable in vitro model to study hepatic drug disposition. SCRH are maintained between two layers of extracellular matrix. In this configuration, culture periods of 4 days are typically applicable. The aim of the present study was to modify conventional SCRH by applying an additional collagen overlay to prolong the hepatic phenotype in SCRH and thus to extend the applicability of the model.

Methods: The cultures receiving an extra top layer ('SCRH-plus' cultures) were compared with the conventional SCRH by testing the morphology, cell functionality, metabolic capacity and Mrp2-activity.

Results: In the SCRH-plus cultures, cell functionality, evaluated by measuring urea production, was increased from day 5 onwards, compared to conventional cultures. Furthermore, these cells retained the appearance of typical hepatocytes, in contrast with conventional sandwich cultures which showed rapid dedifferentiation. SCRH-plus exhibited significantly improved metabolic clearance mediated by cytochrome P450 3A compared to conventional SCRH whereas UDP-glucuronosyltransferase-mediated metabolism was unaffected. Both conventional SCRH and SCRH-plus showed extensive biliary networks at day 4 of culture. However, from day 4 onwards, a decline in biliary excretion index (BEI) was observed in the conventional SCRH, while BEI values in SCRH-plus cultures did not decrease until day 7.

Discussion: The application of an extra top layer of collagen on the SCRH prolongs their useful life-span to 7 days. Therefore, SCRH-plus provide a beneficial impact toward this in vitro model and will broaden the applications for SCRH in terms of long-term toxicity and metabolism of low turnover compounds. 
Keywords: bile canaliculi, collagen, hepatic drug disposition, methods, sandwich-cultured rat hepatocytes 


\section{ABBREVIATIONS}

AUC, Area Under the Curve; BEI, biliary excretion index; CDF, 5-(6)-carboxy-2', $7^{\prime}$ dichlorofluorescein; CDFDA, 5-(6)-carboxy-2',7'-dichlorofluorescein diacetate; $\mathrm{Cl}_{\text {int }}$, intrinsic clearance; CYP, cytochrome P450 enzyme; DMEM, Dulbecco's modified Eagle's Medium; DMSO, dimethylsulfoxide; ECM, extracellular matrix; FBS, fetal bovine serum; HBSS, Hanks' balanced salt solution; HEPES, 4-2-hydroxyethyl-1-piperazineethanesulfonic acid; k, elimination rate constant; Mrp2, multidrug resistance-associated protein 2; PBS, phosphate buffered saline; SCH, sandwich-cultured hepatocytes; SCRH, sandwich-cultured rat hepatocytes; Ugt1, uridine 5'-diphospho-glucuronosyltransferase; WEM, Williams' E medium. 


\section{INTRODUCTION}

Sandwich-cultured (rat) hepatocytes $(\mathrm{SC}(\mathrm{R}) \mathrm{H})$ are widely used to evaluate hepatic drug disposition (De Bruyn et al., 2013). This in vitro tool consists of hepatocytes cultured between two layers of extracellular matrix (ECM). In this configuration, extensive cell-cell and cellECM contacts are formed resulting in an in vivo-like environment. Consequently, the phenotypic expression profile of primary (rat) hepatocytes is maintained for a longer period of time compared to hepatocytes cultured on a single layer of collagen (Dunn et al., 1989; Richert et al., 2002).

In 1989, Dunn et al. first demonstrated the maintenance of hepatocytes in sandwich configuration. In addition, they observed that rat hepatocytes cultured on a single layer of collagen had the ability to retrieve lost functions (e.g. secretion of albumin, bile acids and urea) when a second layer of ECM was applied one week later (Dunn et al., 1991). Ultimately, hepatocytes in a sandwich culture repolarize and reestablish intact biliary networks (Dunn et al., 1991; Hoffmaster et al., 2004; LeCluyse, Audus, \& Hochman, 1994; Liu et al., 1998) Although the decline of phase I, i.e. cytochrome P450 (CYP) activity could not be counteracted in SCRH compared to a single layer collagen configuration. SCRH do allow evaluation of all four phases of the drug elimination process (including uptake, phase I metabolism, phase II metabolism and biliary excretion) (Swift, Pfeifer, \& Brouwer, 2010). For these reasons, SCRH constitute an invaluable in vitro tool with a close comparison to the in vivo situation.

Commonly used ECMs in SCRH are collagen type I (extracted from rat tail tendons) and Matrigel $^{\mathrm{TM}}$ (a protein mixture secreted by mouse sarcoma cells and composed of laminin, type IV collagen and other ECM proteins) (LeCluyse et al., 1996; Richert et al., 2002). Other ECMs evaluated include laminin, fibronectin and ECM hydrogels (Bissell et al., 1986; Skardal et al., 2012). Rat hepatocytes demonstrated a higher affinity for collagen than for 
fibronectin and laminin. Moreover, cell morphology was better maintained (Bissell et al., 1986). However, the composition of the ECM does not appear to be crucial for the expression of CYP enzymes and maintenance of hepatocyte polarity in SCRH (Hamilton et al., 2001; Moghe et al., 1996).

Dedifferentiation of rat hepatocytes maintained in sandwich configuration occurs after 4 days resulting in a limited period of time during which these cultures can be used (LeCluyse et al., 1996; LeCluyse et al., 2012). Consequently, numerous optimization strategies for SCRH have been investigated (e.g. seeding density, culture media and ECM compositions) to enhance the expression and functionality of transport proteins and metabolizing enzymes, and to establish a longer maintenance of hepatocytes in culture (Annaert et al., 2001; De Bruyn et al., 2013; Kern et al., 1997). In fact, Tuschl et al. have shown to preserve the liver-specific functions of SCRH for 10 days when incubating with serum-free medium and such optimizing the sandwich model. Thus, the importance of serum-free conditions have been demonstrated, while the possible benefits of ECM are not clearly shown yet (Tuschl et al., 2009).

In the present study, SCRH were modified to obtain cultures that can be maintained for a longer period of time by applying an additional collagen top layer on day 1 or on day 3 of culture time and such to obtain SCRH-plus. Morphology, biochemical functionality (urea production), hepatic metabolism and biliary excretion were assessed and compared between conventional SCRH and SCRH-plus. 


\section{MATERIALS AND METHODS}

\subsection{Reagents}

ITS+TM Premix was purchased from BD Biosciences (Erembodegem, Belgium). 4-2hydroxyethyl-1-piperazineethanesulfonic acid (HEPES) was obtained from MP Biochemical (Illkirch, France). Williams' E Medium (WEM), L-glutamine, penicillin-streptomycin mixture (containing 10,000 IU/mL potassium penicillin and $10,000 \mu \mathrm{g} / \mathrm{mL}$ streptomycin sulfate), fetal bovine serum (FBS), Hanks' balanced salt solution (HBSS), phosphate buffered saline (PBS), and Trypan blue solution $(0.4 \%)$ were acquired from Lonza Westburg BV (Leusden, The Netherlands). Recombinant human insulin, verapamil, telmisartan, urea, diacetylmonoxime, thiosemicarbazide, iron (III) chloride hexahydrate, orthophosphoric acid, ornithine, collagenase type IV (from Clostridium histolyticum), dexamethasone, Dulbecco's modified Eagle's Medium 10 x (DMEM), Sirius Red (Direct Red 80), picric acid solution and 5-(6)-carboxy-2',7'-dichlorofluorescein diacetate (CDFDA) were purchased from SigmaAldrich (Diegem, Belgium). Ammonium chloride was obtained from UCB (Brussels, Belgium). Sulfuric acid (95- $97 \%)$ and sodium bicarbonate $\left(\mathrm{NaHCO}_{3}\right)$ were purchased from Chem-Lab NV (Zedelgem, Belgium). Sodium hydroxide $(\mathrm{NaOH})$ was obtained from Merck KGaA (Darmstadt, Germany). Leibovitz medium (L-15) was acquired from Invitrogen Corporation (Carlsbad, USA). Dimethyl sulfoxide (DMSO), glucose and Triton X-100 were obtained from Acros Organics (Geel, Belgium). Sodium acetate trihydrate, acetic acid, methanol and acetonitrile were purchased from VWR International (Leuven, Belgium). 24and 48-well sterile cell culture plates were acquired from Greiner Bio-One BVBA (Wemmel, Belgium). Thermostable 96-well plates were kindly provided by Greiner Bio-One BVBA (Wemmel, Belgium). Collagen was prepared in-house from rat tails according to established procedures. 


\subsection{Animals}

Male Wistar rats, weighing $170-200$ g, were housed in the Central Animal Facilities from KU Leuven, according to the Belgium and European laws, guidelines and policies for animal experiments, housing and care. Rats were maintained in a $12 \mathrm{~h}$ light-dark cycle with free access to water and standard rat/mouse maintenance food (ssniff Spezialdiäten $\mathrm{GmbH}$, Germany). The institutional Ethical committee for Animal Experimentation granted approval for these studies.

\subsection{Hepatocyte isolation from rat liver}

Hepatocytes were isolated from male Wistar rats, using a two-step collagenase perfusion method, as described previously (Annaert et al., 2001), without addition of a trypsin inhibitor. The procedure was performed under full anesthesia induced with a mixture of xylazine (24 $\mathrm{mg} / \mathrm{kg})$ and ketamine $(120 \mathrm{mg} / \mathrm{kg})$ given by intraperitoneal injection. After isolation, cells were centrifuged $(50 \mathrm{~g})$ for $3 \mathrm{~min}$ at $4{ }^{\circ} \mathrm{C}$ and the pellet was re-suspended in WEM containing $5 \%$ FBS (v/v), $2 \mathrm{mM}$ L-glutamine, $100 \mathrm{IU} / \mathrm{mL}$ penicillin, and $100 \mu \mathrm{g} / \mathrm{mL}$ streptomycin. Hepatocytes were counted with a hemocytometer and cell viability was determined using Trypan blue. Subsequently, cells were diluted to a final concentration of $1 \times 10^{6}$ cells $/ \mathrm{mL}$ in day-0 medium (WEM containing $5 \%$ FBS (v/v), $2 \mathrm{mM} \mathrm{L-glutamine,} 100 \mathrm{IU} / \mathrm{mL}$ penicillin, and $100 \mu \mathrm{g} / \mathrm{mL}$ streptomycin, $1 \mu \mathrm{M}$ dexamethasone and $4 \mu \mathrm{g} / \mathrm{mL}$ insulin). Freshly-isolated hepatocytes used in following experiments had a viability of at least $88 \%$.

\subsection{Sandwich-cultured rat hepatocytes (referred to as "conventional SCRH")}

24-well sterile culture plates were coated with ice-cold neutralized collagen solution, prepared by neutralizing a mixture of 4 parts of rat-tail collagen $(1.5 \mathrm{mg} / \mathrm{mL}, \mathrm{pH} 7.4), 3$ parts deionized 
water, 1 part of DMEM $10 \mathrm{x}$ and 2 parts of $0.2 \mathrm{~N} \mathrm{NaOH}$, and were placed overnight at $37^{\circ} \mathrm{C}$ in a humidified incubator with $5 \% \mathrm{CO}_{2}$ (Binder $\mathrm{CO}_{2}$ incubator, Binder $\left.\mathrm{GmbH}\right)$. Before seeding, each well was hydrated with $500 \mu \mathrm{L}$ PBS at $37^{\circ} \mathrm{C}$. 48 -well sterile culture plates were coated with $50 \mu \mathrm{g} / \mathrm{mL}$ rigid collagen diluted in $0.02 \mathrm{~N}$ acetic acid (250 $\mu \mathrm{L} /$ well) and were placed overnight at $37{ }^{\circ} \mathrm{C}$ in a humidified incubator with $5 \% \mathrm{CO}_{2}$. Immediately before seeding, the plates were washed twice with PBS at $37{ }^{\circ} \mathrm{C}$. Hepatocytes were seeded at a density of 200000 cells/well and 400000 cells/well in 48- and 24-well culture plates, respectively, as described previously (Keemink, Oorts, \& Annaert, 2014). After incubating the cells at $37{ }^{\circ} \mathrm{C}$ in a humidified incubator with $5 \% \mathrm{CO}_{2}$ for $1-2 \mathrm{~h}$, unattached cells were removed by shaking the plates and immediately aspirating the medium. To obtain a sandwich configuration, the cells were overlaid with 30 or $50 \mu \mathrm{L}$ ice-cold neutralized rat tail collagen solution (1.5 mg/mL, $\mathrm{pH} 7.4)$ in 48- and 24-well culture plates, respectively. After $1 \mathrm{~h}$, prewarmed day- 0 medium was added onto the cultures which were kept in a humidified incubator with $5 \% \mathrm{CO}_{2}$. The medium was changed daily with completed culture medium consisting of WEM supplemented with $1 \%(\mathrm{v} / \mathrm{v})$ ITS+TM Premix, 2 mM L-glutamine, 100 $\mathrm{IU} / \mathrm{mL}$ penicillin, $100 \mu \mathrm{g} / \mathrm{mL}$ streptomycin, and $0.1 \mu \mathrm{M}$ dexamethasone. Throughout this study, conditions where cells were overlaid once (on day 0) are called "single top layer (STL)".

\subsection{Additional collagen overlay in SCRH (referred to as "SCRH-plus")}

On day 1 or day 3 of the cultures, medium was aspirated from SCRH and cells were overlaid with an additional top layer of ice-cold neutralized rat tail collagen solution $(1.5 \mathrm{mg} / \mathrm{mL}, \mathrm{pH}$ 7.4), and kept in a humidified incubator for $1 \mathrm{~h}$, as described above. Subsequently, completed culture medium was added onto the cells. Throughout this study, SCRH overlaid with a 
second collagen layer at day 1 or day 3 of the culture, are addressed to as "double top layer 1 (DTL1)" and "double top layer 3 (DTL3)", respectively.

\subsection{Determination of urea production}

The capacity of the hepatocytes to convert ammonia to urea was used to assess the overall biochemical functionality of rat hepatocytes (Chatterjee et al., 2014; Oorts, Richert, \& Annaert, 2015). Briefly, the cells were washed twice with standard buffer (HBSS containing $10 \mathrm{mM}$ HEPES, $\mathrm{pH} 7.4$ ) at $37^{\circ} \mathrm{C}$ and incubated with incubation buffer (HBSS containing 10 $\mathrm{mM}$ HEPES, $2 \mathrm{mM}$ glutamine, $10 \mathrm{mM}$ ammonium chloride and $3 \mathrm{mM}$ ornithine) (250 $\mu \mathrm{L} /$ well for 24 -well plates; $125 \mu \mathrm{L} /$ well for 48 -well plates) for $1 \mathrm{~h}$ at $37{ }^{\circ} \mathrm{C}$ in a humidified incubator with $5 \% \mathrm{CO}_{2}$. Subsequently, $60 \mu \mathrm{L}$ of the incubation buffer/well was mixed with $240 \mu \mathrm{L}$ of color reagent in a 96-well thermostable plate. The mixture of color reagent and incubate was heated at $85{ }^{\circ} \mathrm{C}$ for 20 min and cooled down by keeping the plate at $4{ }^{\circ} \mathrm{C}$ for 10 min. Absorbance was measured at $525 \mathrm{~nm}$ using a Tecan Infinite M200 plate reader (Tecan Group Ltd., Männedorf, Austria). The results of the urea production in the SCRH-plus are expressed as fold change versus the conventional SCRH.

\subsection{Hepatic metabolism}

Metabolism studies were performed in SCRH (-plus), cultured in 24-well culture plates in a shaking incubator $\left(300 \mathrm{rpm}, 37^{\circ} \mathrm{C}\right)$. Verapamil and telmisartan solutions were prepared in 90 \% L-15 medium, supplemented with glutamine (3.6 mM), glucose (9.9 mM), HEPES (9 mM) and $\mathrm{NaHCO}_{3}(3.6 \mathrm{mM})$. Medium was aspirated from the SCRH, and cells were incubated with $250 \mu \mathrm{L}$ of verapamil $(5 \mu \mathrm{M})$ or telmisartan $(5 \mu \mathrm{M})$ at $37^{\circ} \mathrm{C}$. At different time points $(0$, 20, 40 and $60 \mathrm{~min}$ ), $500 \mu \mathrm{L}$ methanol was added to the wells to quench the reaction. After 15 
minutes, aliquots were taken from each well and stored at $-20{ }^{\circ} \mathrm{C}$ until analysis. After each experiment, the mean protein content/well was determined using BCA Protein assay kit (Pierce Chemical, Rockford, Illinois).

Intrinsic clearance $\left(\mathrm{Cl}_{\text {int }}\right)$ was assessed with the 'in vitro $\mathrm{t}_{1 / 2}$ method'(Obach, 1999). Briefly, concentrations were converted to the percentage of the drug remaining. The slope of the linear regression from the log percentage remaining versus incubation time was used to calculate the elimination rate constant $(\mathrm{k})$ and the in vitro $\mathrm{t}_{1 / 2}$. Finally, $\mathrm{Cl}_{\mathrm{int}}$ was determined according to:

$$
C l_{\text {int }, \text { met }, \text { mic }}=\frac{0.693}{t_{1 / 2(\min )}} \times \frac{\text { incubation volume }(\mu L)}{\text { cell density }(\text { million cells })}
$$

Metabolic data were normalized for the cell density (1.19 million cells/mg protein) during the incubation periods and expressed as $\mu \mathrm{L} / \mathrm{min} / \mathrm{million}$ cells.

\subsection{HPLC analysis}

Verapamil: Verapamil was analyzed using a Waters 600 multisolvent delivery system, a Waters 717 plus autosampler with temperature control $\left(21^{\circ} \mathrm{C}\right)$, a Waters 2475 fluorescence detector (ex/em: 230/312 nm) and a Gemini ${ }^{\circledR}$ C18 column $(3 \mu \mathrm{m}, 4.6 \mathrm{~mm} \mathrm{x} 150 \mathrm{~mm}$, Phenomenex, Utrecht, The Netherlands). The mobile phase consisted of $60: 40 \%(\mathrm{v} / \mathrm{v})$ buffer:methanol under isocratic conditions. The buffer was a $25 \mathrm{mM}$ sodium acetate buffer containing $10 \mathrm{mM}$ Triethanolamine ( $\mathrm{pH} 5.5)$. The flow rate was $0.6 \mathrm{~mL} / \mathrm{min}$, the retention time $7.8 \mathrm{~min}$ and the run time $10 \mathrm{~min}$. Peaks were integrated using Empower Pro (Empower 2) software. Accuracy and precision of quality control samples $(0.1,1$ and $10 \mu \mathrm{M})$ was below $10 \%$.

Telmisartan: Telmisartan was analyzed using a Surveyor FL Plus Detector (ex/em 280/360 nm) coupled with a Surveyor HPLC system (Thermo, San Jose, CA, USA) and a Kinetex C18 column (5 $\mathrm{mm}, 150 \mathrm{~mm}$ x $4.6 \mathrm{~mm})$ (Phenomenex, Utrecht, The Netherlands). The mobile 
phase consisted of 25:45:30 \% (v/v) buffer:methanol:acetonitrile and ran at a flow of 0.8 $\mathrm{mL} / \mathrm{min}$ under isocratic conditions. The buffer contained $25 \mathrm{mM}$ sodium acetate buffer $(\mathrm{pH}$ 4.2). The retention time was $4.7 \mathrm{~min}$ and the run time $5.5 \mathrm{~min}$. Peaks were integrated using Chromquest 5.0 software. Accuracy and precision of quality control samples $(0.1,0.5,1 \mu \mathrm{M})$ was below $10 \%$.

\subsection{Light and fluorescence microscopy}

The biliary excretory function of SCRH(-plus) was assessed by quantitative evaluation of 5(6)- carboxy-2',7'-dichlorofluorescein (CDF) excretion in bile canalicular networks via fluorescence microscopy (ex/em 490/520 nm). Briefly, SCRH(-plus) were washed twice with standard buffer at $37{ }^{\circ} \mathrm{C}$ and then pre-incubated with standard buffer for $10 \mathrm{~min}$. Next, hepatocytes were incubated with $4 \mu \mathrm{M}$ CDFDA in standard buffer. After a 10 min incubation at $37{ }^{\circ} \mathrm{C}$, the buffer was aspirated. Hepatocytes and biliary network were imaged (both by fluorescence and light microscopy) with a VisiCam ${ }^{\circledR} 3.0$ camera (VWR International Bvba, Leuven, Belgium), mounted on a Olympus IX70 inverted tissue culture microscope (Olympus Optical Co GMBH, Hamburg, Germany). A monochromator (Polychrome IV; Till Photonics, Oberhausen, Germany) was used to generate the excitation wavelength $(490 \mathrm{~nm})$. For fluorescence microscopy a U-MWIB3 mirror unit was used (long pass emission filter (510 $\mathrm{nm})$; dichroic mirror $(505 \mathrm{~nm})$ ). Image $\mathrm{J}(\mathrm{NIH}$, version 1.48s) was used to select areas representing the bile canaliculi, the intracellular compartment and background areas. Subsequently, fluorescence intensity/pixel of canalicular and intracellular areas was determined taking into account background. Moreover, areas of these compartments were determined as well as the total surface are of the pictures to determine the bile canalicular area and the biliary excretion index (BEI).

The microscopic image analysis-based BEI of CDF was determined according to: 


$$
B E I=\frac{X_{\text {bile }}}{X_{\text {cells }}+X_{\text {bile }}}
$$

with $\mathrm{X}$ the intensity/pixel multiplied by the selected area (pixels).

\subsection{Collagen quantification}

To determine the amount of collagen present in the top layer of SCRH (-plus), culture medium was aspirated and hepatocytes were rinsed twice with PBS. In order to dissolve the collagen top layer, cells were incubated with $0.05 \mathrm{M}$ acetic acid (500 $\mu \mathrm{L} /$ well) for $10 \mathrm{~min}$ at room temperature. After incubation, medium samples $(100 \mu \mathrm{L})$ were taken and transferred to $1.5 \mathrm{~mL}$ Eppendorf tubes. Subsequently, $500 \mu \mathrm{L}$ of Dye solution, containing $0.1 \%(\mathrm{w} / \mathrm{v})$ Sirius red in aqueous solution of picric acid (1.3\% in water) was added. Samples were vortexed and incubated at room temperature for $20 \mathrm{~min}$, after which the tubes were centrifuged for $3 \mathrm{~min}$ at $10621 \mathrm{x} g\left(4^{\circ} \mathrm{C}\right)$. The supernatant was carefully removed and the resulting pellet was resuspended in ultrapure water $(500 \mu \mathrm{L})$. Centrifugation was repeated (10 $621 \mathrm{x} \mathrm{g}$ ) for $3 \mathrm{~min}$ at $4^{\circ} \mathrm{C}$, after which the supernatant was carefully removed. $250 \mu \mathrm{L}$ of extraction buffer $(1: 1 \mathrm{MeOH} / \mathrm{NaOH} 0.1 \mathrm{~N})$ was added to the pellet and the tubes were vortexed until the pellet was completely dissolved. The samples were diluted (5x) using Extraction buffer and $200 \mu \mathrm{L}$ of each sample was transferred to a 96-well plate. Finally, absorbance was measured at 540nm using a Tecan Infinite M200 plate reader.

\subsection{Statistics}

A $95 \%$ confidence interval was calculated to determine whether the SCRH-plus resulted in a statistically significant improvement of cell functionality.

Statistical analysis was performed using a two-tailed unpaired t-test to evaluate differences between two groups, or one-way ANOVA followed by a Dunnett's test to compare DTL cultures to STL (control) cultures. P-values less than 0.05 were considered statistically significant. 


\section{RESULTS}

\subsection{Biochemical functionality}

A urea assay was performed to quantify the level of biochemical functionality of the hepatocytes. An additional layer of collagen, applied either on day 1 or 3, resulted in a significantly higher urea production compared to conventional sandwich cultures. This was reflected by a ratio greater than 1 in both 24- and 48-well plates after 5, 6 and 7 days in culture (Figure 1). Moreover, higher urea production was already observed on day 3 in cells that received an extra collagen top layer on day 1 in 24-well plates (Figure 1A).

\subsection{Morphology}

Figure 2 shows that conventional SCRH exhibited extensive dedifferentiation after 5 days of culture. On the contrary, hepatocytes overlaid with a second top layer of collagen on day 1 or 3 were morphologically similar to each other and exhibited a uniform and polygonal morphology, until day 7. Moreover, the extra collagen overlay resulted in more extensive formation of distinct (and functional) bile canaliculi compared to conventional SCRH. Table 1 shows the area covered with bile canaliculi for the different conditions. The SCRH-plus cultures exhibited a more extensive network of bile canaliculi than the conventional SCRH. The surface area represented by the bile canaliculi in these cultures remained constant whereas the area corresponding to bile canaliculi in conventional sandwich cultures tended to decrease over time (between day 5 and 7). 


\subsection{Metabolism}

The activity of phase I enzymes was evaluated using the CYP3A-substrate verapamil. $\mathrm{Cl}_{\text {int }}$ values determined in hepatocytes overlaid with an extra top layer of collagen on day 1 were significantly higher than values obtained in conventional SCRH on day 6 and 7 (Figure 3). Moreover, the extra overlay on day 3 resulted in higher $\mathrm{Cl}_{\text {int }}$ values compared to the conventional sandwich-cultures on day 5, 6 and 7. Higher $\mathrm{Cl}_{\text {int }}$ values indicate better maintenance of CYP3A activity.

Metabolism of the uridine 5'-diphospho-glucuronosyltransferase (Ugt1)-substrate telmisartan was assessed to investigate the activity of a phase II enzyme. On day 4, Ugt1 activity in conventional SCRH was slightly higher. On day 5, 6 and 7 Ugt1 was consistent between different conditions (Figure 4).

\subsection{Biliary excretion}

CDF was chosen as a substrate to assess the multidrug resistance-associated protein 2 (Mrp2; $A b c c 2)$ mediated biliary excretion. After 4 days in culture, the mean BEI ( \pm SEM) in conventional cultures was $47 \pm 6 \%$. When an extra overlay of collagen was applied on day 1 or 3, mean BEI values ( \pm SEM) on day 4 were $58 \pm 4 \%$ and $61 \pm 2 \%$, respectively (Table 2 ). From day 5 onwards, BEI-values were significantly higher in cultures overlaid with a second top layer of collagen compared to the conventional SCRH (Table 2, Supplemental figure 1). Over time in culture, BEI values for all conditions remained constant between days 4 and 6 and decreased on day 7 . 


\subsection{Collagen quantification}

Amounts of collagen in the top layer were quantified on day 2 to 7 for the SCRH-plus cultures with the extra overlay applied on day 1 and on day 4 to 7 for the SCRH-plus cultures with the extra overlay applied on day 3 (Figure 5). Although top layers of SCRH-plus cultures tended to contain more collagen, no significant difference was observed between the AUC values calculated based on the absorbance versus culture time curves, as shown in Table 3. If we compare the collagen amount on the individual days, only the top layer of the SCRH-plus that received an additional top layer at day-3 (DTL3) contained significantly more collagen as compared to the conventional SCRH (STL1). 


\section{DISCUSSION}

Rat hepatocytes cultured in sandwich configuration have been shown to preserve some hepatocyte-specific functions including phase I and II metabolism as well as transportermediated drug uptake and excretion. Therefore, SCRH are a widely used in vitro tool for the evaluation of hepatobiliary drug transport and metabolism. However, with this model, a relatively short culture time of maximally 4 days is commonly applicable (LeCluyse et al., 1994). Nevertheless, a few studies have succeeded to significantly prolong the maintenance of the hepatocyte phenotype. In particular, Tuschl et al. have described the use of serum-free culture conditions to significantly prolong the liver-like properties of sandwich-cultured rat hepatocytes (Tuschl et al., 2009). While the latter study also mentioned the importance of the ECM to maintain the hepatic phenotype, this was not specifically investigated. Therefore, the present study specifically explored the possible benefit of applying an additional layer of collagen on top of conventional sandwich cultures in order to maintain functional rat hepatocytes for up to 7 days (as opposed to the standard culture time of 4 days).

As compared to conventionally cultured SCRH, our results revealed improved cell functionality, as measured by the capability of the hepatocytes to convert ammonia to urea, in cultures overlaid with an additional top layer of collagen. Two days after the addition of an extra collagen top layer on day $1, \mathrm{SCRH}$ in 24 -well plates already showed a significantly higher urea production. Furthermore, from day 5 onwards, mean ratios of urea production in cells with a double top layer as opposed to a single top layer were significantly higher than 1 , irrespective of the day that the extra overlay was applied or the size of the well plate (Figure 1). The relatively high urea production in the SCRH-plus is predominantly due to a fast decline in urea production over time in the conventional sandwich cultures. On the contrary, the urea production in cultures with an extra overlay was stable. A more pronounced 
reduction in urea production in SCRH in 48-well plates explained the high ratio obtained on day 7 in these culture plates.

Moreover, the morphology of hepatocytes in the SCRH-plus was superior to conventional SCRH. Conventional sandwich cultures displayed dedifferentiation after 5 days, which resulted in extensive spreading of the cells. Hepatocytes also started to detach, and consequently died. On the contrary, in the SCRH-plus, polygonal morphology and extensive bile canalicular networks are maintained up to day 7 (Table 2; Figure 2). It has been shown previously that hepatocytes cultured in monolayers lose their morphology after $24-48 \mathrm{~h}$ and that this deterioration was delayed in sandwich cultures where hepatocytes could be maintained for 3 - 4 days (LeCluyse et al., 1994; Richert et al., 2002). In the present study, we show that the extra collagen layer on top of the SCRH delays that process even more, thus extending the useful culture period with $3-4$ extra days. A similar result was also observed when SCRH were re-overlayed with Matrigel $^{\mathrm{TM}}$ (Germano et al., 2015).

CYP3A enzymes are involved in the metabolism of approximately $50 \%$ of all small molecule drugs (Zuber, Anzenbacherová, \& Anzenbacher, 2002). Therefore, CYP3A-mediated metabolism was evaluated in conventional SCRH and SCRH-plus using verapamil as a substrate. From day 5 onwards, cultures with a double collagen top layer showed improved metabolic clearance of verapamil, compared to SCRH with a single top layer. Kern et al. previously reported an increase in CYP3A1 activity between day 3 and 10 in sandwich cultures (Kern et al., 1997). However, in the present study, an increase has only been observed between day 4 and 7 in SCRH-plus (Figure 3). This inconsistency might be explained by the involvement of CYP3A2 in verapamil metabolism. As opposed to CYP3A1, no information regarding activity of this enzyme in function of culture time could be found in the literature. In addition, no major effect of SCRH-plus was found regarding Ugt-1 mediated metabolism, which, over time, remains constant in all conditions (Figure 4). This is in 
accordance with previous reports showing that phase II is better maintained in culture than phase I, and that as a consequence a phase I/phase II imbalance occurs in hepatocytes during time of culture in both monolayer and sandwich cultures (De Bruyn et al., 2013).

Canalicular localization of Mrp2 increases in function of culture time resulting in increasing biliary excretion of CDF between day 0 and 4 (Zhang et al., 2005). However, after day 4, we observed a decline in BEI in conventional sandwich cultures. On the contrary, in the SCRHplus, BEI values did not decrease until day 7. Additionally, BEI values in these cultures were significantly increased compared to the conventional SCRH from day 5 onwards, indicating an improved functionality of canalicular transporters in SCRH-plus (Table 2). The imagebased BEI analysis determined in this study resulted in higher values compared to the more commonly used indirect methods (Zhang et al., 2005). Different experimental systems could explain this difference which has also been observed previously for tauro-nor-THCA-24-DBD (De Bruyn et al., 2014). An implicit advantage of the image-based approach is that any contribution of fluorescence intensity in dead cells was excluded with the present method based on ImageJ-based processing of the fluorescence microscopic images. Another major advantage of the non-destructive nature of this image-based procedure used for quantitative determination of CDF was that it allows 'recycling' the cells, meaning that the hepatocyte cultures can be used more efficiently in subsequent experiments and/or additional endpoints. Taken together, the results confirm a prolonged maintenance of functional SCRH following an additional collagen overlay. An additional collagen overlay could possibly lead to an improved rigidity of the collagen upon culture time, resulting in the maintenance of the embedment of the cells in the collagen layer. In addition, these results are in accordance with the recent observations of Germano et al. that maintenance of rat hepatocyte morphology as well as the excretory function in collagen-Matrigel ${ }^{\mathrm{TM}}$ sandwich culture was extended following extra overlays of SCRH with Matrigel every 3-4 days (Germano et al., 2015). 
However, the present study also investigated metabolic capacity of double overlaid cultures and deterioration in function of culture time as compared to the control situation on day 4 . In addition, a prolonged maintenance of both morphology and functionality of SCRH were found. Similar observations were made with human hepatocytes in collagen-Matrigel ${ }^{\mathrm{TM}}$ sandwich culture conditions (Parmentier et al., 2013, 2017).

It is recognized that the presence of collagen maintains an "in vivo-like" environment for the hepatocytes enabling cell-ECM contacts and therefore maintenance of liver-specific gene expression (Dunn et al., 1991; LeCluyse et al., 1996).

In addition, it has recently been shown that collagen secretion in flow-cultures of hepatocytes explains the preservation of hepatic functions in these cultures (Hegde et al., 2014). Indeed, it is a well-known fact that hepatocytes, both in vivo and in vitro, have the capability of synthesizing collagen proportionally to proline levels present in the medium (Lee et al., 1993). However, stellate cells are the major contributor of collagen synthesis in vivo (Yi \& Jeong, 2013). Since stellate cells are absent in SCRH and collagen secretion in those static cultures is restricted due to a limited availability of proline, we demonstrated that the additional top layer of collagen resulted in slightly larger quantities of collagen in the cultures, ensuring the presence of sufficient attachment sites for cells to ECM components. Therefore, the increased amount of collagen present in the SCRH-plus results in the maintenance of differentiated hepatocytes.

The SCRH-plus with a prolonged overall performance provides an in vitro tool that allows extended incubation periods. The model thus appears appropriate to explore long-term hepatotoxicity, as previously reported with human hepatocytes when re-overlay consisted of Matrigel $^{\mathrm{TM}}$ (Parmentier et al., 2013, 2017). Additionally, this configuration enables clearance predictions for poorly metabolized compounds. Short incubation periods imposed by suspended hepatocytes have partly circumvented using conventional SCRH for these 
applications (Ansede et al., 2010; Chatterjee et al., 2014; Oorts et al., 2015; Treijtel et al., 2004). However, the proposed modification of these cultures with the extra top layer of collagen permits incubation periods to up to 7 days. In view of the previously described advantage of serum-free culture to extend the maintenance of SCRH to 10 days (Tuschl et al., 2009), it would certainly be interesting to subsequently explore the combined benefit of serum-free conditions and additional collagen overlay.

Nowadays, research in the development and optimization of co-cultures (e.g. cultures of hepatocytes and non-parenchymal cells) and 3D-cultures is expanding. These models focus on the in vivo resemblance of the cells, but are often restricted by their complexity and timeconsuming techniques. The present study describes a relatively easy practical procedure resulting in significantly improved hepatocyte culture quality. Therefore, the addition of ECM during cultivation could have an added value to co-cultures and 3D-cultures.

To summarize, we describe a modified method of SCRH which allows the long-term maintenance of rat hepatocytes in sandwich configuration. The additional layer of collagen on top of the cells, applied on day 1 or day 3 , resulted in the preservation of the differentiated cell type as assessed by cellular morphology, biochemical functionality, a better balanced phase I and II by maintenance of phase I CYP3A activity as well as functional biliary excretion until day 7. SCRH-plus provide a beneficial impact toward this in vitro model and will broaden the applications for SCRH in terms of long-term toxicity and metabolism of low turnover compounds. 


\section{ACKNOWLEDGEMENTS}

Financial support for this research was provided by the Institute for the promotion of Innovation by Science and Technology in Flanders (IWT) under Grant SBO-IWT.

\section{AUTHOR DISCLOSURE STATEMENT}

The authors have no conflicts of interest.

\section{REFERENCES}

Annaert, P. P., Turncliff, R. Z., Booth, C. L., Thakker, D. R., \& Brouwer, K. I. M. L. R. (2001). P-glycoprotein-mediated in vitro biliary excretion in sandwich-cultured rat hepatocytes. Drug Metabolism and Disposition: The Biological Fate of Chemicals, 29(10), 1277-83.

Ansede, J. H., Smith, W. R., Perry, C. H., St, R. L., Iii, C., Brouwer, K. R., ... Brouwer, K. R. (2010). An in vitro assay to assess transporter-based cholestatic hepatotoxicity using sandwich-cultured rat hepatocytes. Drug Metabolism and Disposition: The Biological Fate of Chemicals, 38(2), 276-280. http://doi.org/10.1124/dmd.109.028407

Bissell, D. M., Stamatoglou, S. C., Nermut, M. V, \& Hughes, R. C. (1986). Interactions of rat hepatocytes with type IV collagen, fibronectin and laminin matrices. Distinct matrixcontrolled modes of attachment and spreading. European Journal of Cell Biology, 40(1), 728.

Chatterjee, S., Richert, L., Augustijns, P., \& Annaert, P. (2014). Hepatocyte-based in vitro model for assessment of drug-induced cholestasis. Toxicology and Applied Pharmacology, 274(1), 124-36. http://doi.org/10.1016/j.taap.2013.10.032

De Bruyn, T., Chatterjee, S., Fattah, S., Keemink, J., Nicolaï, J., Augustijns, P., \& Annaert, P. 
(2013). Sandwich-cultured hepatocytes: utility for in vitro exploration of hepatobiliary drug disposition and drug-induced hepatotoxicity. Expert Opinion on Drug Metabolism \& Toxicology, 9(5), 589-616. http://doi.org/10.1517/17425255.2013.773973

De Bruyn, T., Sempels, W., Snoeys, J. A. N., Holmstock, N., Chatterjee, S., Stieger, B., ... Annaert, P. (2014). Confocal imaging with a fluorescent bile acid analogue closely mimicking hepatic taurocholate disposition. Journal of Pharmaceutical Sciences, 103(6), 1872-81. http://doi.org/10.1002/jps.23933

Dunn, J. C., Tompkins, R. G., \& Yarmush, M. L. (1991). Long-term in vitro function of adult hepatocytes in a collagen sandwich configuration. Biotechnology Progress, 7(3), 237-45. http://doi.org/10.1021/bp00009a007

Dunn, J. C., Yarmush, M. L., Koebe, H. G., \& Tompkins, R. G. (1989). Hepatocyte function and extracellular matrix geometry: long-term culture in a sandwich configuration. FASEB Journal : Official Publication of the Federation of American Societies for Experimental Biology, 3(2), 174-7.

Germano, D., Uteng, M., Pognan, F., Chibout, S.-D., \& Wolf, A. (2015). Determination of liver specific toxicities in rat hepatocytes by high content imaging during 2-week multiple treatment. Toxicology in Vitro, 30(1), 79-94. http://doi.org/10.1016/j.tiv.2014.05.009

Hamilton, G. A., Jolley, S. L., Gilbert, D., Coon, D. J., Barros, S., \& LeCluyse, E. L. (2001). Regulation of cell morphology and cytochrome P450 expression in human hepatocytes by extracellular matrix and cell-cell interactions. Cell and Tissue Research, 306(1), 85-99.

Hegde, M., Jindal, R., Bhushan, A., Bale, S. S., McCarty, W. J., Golberg, I., ... Yarmush, M. L. (2014). Dynamic interplay of flow and collagen stabilizes primary hepatocytes culture in a microfluidic platform. Lab on a Chip, 14(12), 2033-9. http://doi.org/10.1039/c4lc00071d 
Hoffmaster, K. A., Turncliff, R. Z., LeCluyse, E. L., Kim, R. B., Meier, P. J., \& Brouwer, K. L. R. (2004). P-glycoprotein expression, localization, and function in sandwich-cultured primary rat and human hepatocytes: relevance to the hepatobiliary disposition of a model opioid peptide. Pharmaceutical Research, 21(7), 1294-302.

Keemink, J., Oorts, M., \& Annaert, P. (2014). Primary hepatocytes in sandwich culture. Methods in Molecular Biology (Vol. 1250). http://doi.org/10.1007/978-1-4939-2074-7_12

Kern, A., Bader, A., Pichlmayr, R., \& Sewing, K. F. (1997). Drug metabolism in hepatocyte sandwich cultures of rats and humans. Biochemical Pharmacology, 54(7), 761-72.

LeCluyse, E. L., Audus, K. L., \& Hochman, J. H. (1994). Formation of extensive canalicular networks by rat hepatocytes cultured in collagen-sandwich configuration. The American Journal of Physiology, 266(6 Pt 1), C1764-74.

LeCluyse, E. L., Bullock, P. L., Parkinson, A., \& Hochman, J. H. (1996). Cultured rat hepatocytes. Pharmaceutical Biotechnology, 8, 121-59.

LeCluyse, E. L., Witek, R. P., Andersen, M. E., \& Powers, M. J. (2012). Organotypic liver culture models: meeting current challenges in toxicity testing. Critical Reviews in Toxicology, 42(6), 501-48. http://doi.org/10.3109/10408444.2012.682115

Lee, J., Morgan, J. R., Tompkins, R. G., \& Yarmush, M. L. (1993). Proline-mediated enhancement of hepatocyte function in a collagen gel sandwich culture configuration. FASEB Journal : Official Publication of the Federation of American Societies for Experimental Biology, 7(6), 586-91.

Liu, X., Brouwer, K. R. L., Gan, L. S., Brouwer, K. R. L., Stieger, B., Meier, P. J., ... LeCluyse, E. L. (1998). Partial maintenance of taurocholate uptake by adult rat hepatocytes cultured in a collagen sandwich configuration. Pharmaceutical Research, 15(10), 1533-9. 
Moghe, P. V, Berthiaume, F., Ezzell, R. M., Toner, M., Tompkins, R. G., \& Yarmush, M. L. (1996). Culture matrix configuration and composition in the maintenance of hepatocyte polarity and function. Biomaterials, 17(3), 373-85.

Obach, R. S. (1999). Prediction of human clearance of twenty-nine drugs from hepatic microsomal intrinsic clearance data: An examination of in vitro half-life approach and nonspecific binding to microsomes. Drug Metabolism and Disposition: The Biological Fate of Chemicals, 27(11), 1350-9.

Oorts, M., Richert, L., \& Annaert, P. (2015). Drug-induced cholestasis detection in cryopreserved rat hepatocytes in sandwich culture. Journal of Pharmacological and Toxicological Methods, 73, 63-71. http://doi.org/10.1016/j.vascn.2015.03.002

Parmentier, C., Couttet, P., Wolf, A., Zaccharias, T., Heyd, B., Bachellier, P., ... Richert, L. (2017). Evaluation of transcriptomic signature as a valuable tool to study drug-induced cholestasis in primary human hepatocytes. Archives of Toxicology. http://doi.org/10.1007/s00204-017-1930-0

Parmentier, C., Truisi, G. L., Moenks, K., Stanzel, S., Lukas, A., Kopp-Schneider, A., ... Richert, L. (2013). Transcriptomic hepatotoxicity signature of chlorpromazine after short- and long-term exposure in primary human sandwich cultures. Drug Metabolism and Disposition: The Biological Fate of Chemicals, 41(10), 1835-42. http://doi.org/10.1124/dmd.113.052415 Richert, L., Binda, D., Hamilton, G., Viollon-Abadie, C., Alexandre, E., Bigot-Lasserre, D., ... LeCluyse, E. (2002). Evaluation of the effect of culture configuration on morphology, survival time, antioxidant status and metabolic capacities of cultured rat hepatocytes. Toxicology in Vitro : An International Journal Published in Association with BIBRA, 16(1), 89-99. 
Skardal, A., Smith, L., Bharadwaj, S., Atala, A., Soker, S., \& Zhang, Y. (2012). Tissue specific synthetic ECM hydrogels for 3-D in vitro maintenance of hepatocyte function. Biomaterials, 33(18), 4565-75. http://doi.org/10.1016/j.biomaterials.2012.03.034

Swift, B., Pfeifer, N. D., \& Brouwer, K. L. R. (2010). Sandwich-cultured hepatocytes: an in vitro model to evaluate hepatobiliary transporter-based drug interactions and hepatotoxicity. Drug Metabolism Reviews, 42(3), 446-71. http://doi.org/10.3109/03602530903491881

Treijtel, N., Barendregt, A., Freidig, A. P., Blaauboer, B. J., \& van Eijkeren, J. C. H. (2004). Modeling the in vitro intrinsic clearance of the slowly metabolized compound tolbutamide determined in sandwich-cultured rat hepatocytes. Drug Metabolism and Disposition: The Biological Fate of Chemicals, 32(8), 884-91.

Tuschl, G., Hrach, J., Walter, Y., Hewitt, P. G., \& Mueller, S. O. (2009). Serum-free collagen sandwich cultures of adult rat hepatocytes maintain liver-like properties long term: a valuable model for in vitro toxicity and drug-drug interaction studies. Chemico-Biological Interactions, 181(1), 124-37. http://doi.org/10.1016/j.cbi.2009.05.015

Yi, H.-S., \& Jeong, W.-I. (2013). Interaction of hepatic stellate cells with diverse types of immune cells: Foe or friend? Journal of Gastroenterology and Hepatology, 28, 99-104. http://doi.org/10.1111/jgh.12017

Zhang, P., Tian, X., Chandra, P., \& Brouwer, K. L. R. (2005). Role of glycosylation in trafficking of Mrp2 in sandwich-cultured rat hepatocytes. Molecular Pharmacology, 67(4), 1334-41. http://doi.org/10.1124/mol.104.004481

Zuber, R., Anzenbacherová, E., \& Anzenbacher, P. (2002). Cytochromes P450 and experimental models of drug metabolism. Journal of Cellular and Molecular Medicine, 6(2), 189-98. 


\section{FIGURE LEGENDS}

Figure 1. The mean ratio $\pm 95 \%$ confidence interval of urea production in sandwichcultured rat hepatocytes (SCRH) with an additional top collagen layer on day 1 (DTL1, grey bars) or day 3 (DTL3, white bars) versus SCRH with a single top collagen layer (STL). Urea production was measured in triplicate in 3 batches $(n=3)$ of SCRH seeded in 24-well culture plates (A), and in 48-well culture plate (B).

Figure 2. Morphology of sandwich-cultured rat hepatocytes (SCRH) after a single top layer of collagen (STL) or with an additional top layer at day 1 (DTL1) or day 3 (DTL3). Light microscopic images of SCRH, representative for all batches used during the study (Magnification x 100).

Figure 3. Intrinsic clearance $\left(\mathrm{Cl}_{\mathrm{int}}\right) \pm \mathrm{SD}$ of verapamil in function of culture time in conventional sandwich-cultured rat hepatocytes (SCRH; black bars; STL) or SCRHplus with an additional overlay of collagen applied on day 1 (grey bars; DTL1) or day 3 (white bars; DTL3). The disappearance of verapamil was measured in triplicate in 2 batches of hepatocytes $(n=2)$. Statistical significance between different conditions at each culture time was determined using a one-way ANOVA followed by a Dunnett's test with STL as control $(* \mathrm{p}<0.05 ; * * \mathrm{p}<0.01 ; * * * \mathrm{p}<0.001)$

Figure 4. Intrinsic clearance $\left(\mathrm{Cl}_{\mathrm{int}}\right) \pm \mathrm{SD}$ of telmisartan in function of culture time in conventional sandwich-cultured rat hepatocytes (SCRH; black bars; STL) or SCRHplus with an additional top layer applied on day 1 (greys bars; DTL1) or day 3 (white 
bars; DTL3). The disappearance of telmisartan was measured in triplicate in two batches of hepatocytes in triplicate $(n=2)$. Statistical significance between different conditions at each culture time point was determined using a one-way ANOVA followed by a Dunnett's test for which STL is used as a control $(* \mathrm{p}<0.05 ; * * \mathrm{p}<0.01)$.

Figure 5. Mean ( \pm SEM) amounts of collagen extracted from the top layer of sandwichcultured rat hepatocytes (SCRH) with a single layer of collagen (STL) or with an additional layer applied on: (A) day 1 (DTL1); or (B) day 3 (DTL3). Collagen content in the top layer was determined in triplicate in 3 different rat hepatocyte batches $(n=3)$ seeded in 24-well plates. Statistical significance between STL and DTL cultures at each day was evaluated using a unpaired two-tailed t-test $(* \mathrm{p}<0.05)$. 


\section{SUPPLEMENTAL FIGURES}

Supplemental Figure 1. Biliary excretion of CDF in SCRH after a single top layer of collagen (STL) or with an additional top layer of collagen at day 1 (DTL1) or day 3 (DTL3) at day-7 of culture time. Microscopic images of SCRH, representative for all batches used during the study (Magnification x 100). 
10. TABLES

Table 1. The relative area (\%) in sandwich-cultured rat hepatocytes (SCRH) representing bile canaliculi. Bile canaliculi were visualized with 5-(6)-carboxy-2',7'dichlorofluorescein (CDF). Surface areas were determined using ImageJ. Results represent the mean values ( \pm SEM) obtained from three pictures in each of two hepatocyte cultures. Statistical significance between STL and DTL were measured using a one-way ANOVA followed by a Dunnett's $(* * \mathrm{p}<0.01 ; * * * \mathrm{p}<0.001)$

\section{Relative surface area of the cultures representing bile canaliculi} (as \% of total surface area)

\begin{tabular}{lcccc}
\hline & \multicolumn{1}{c}{ day 4} & \multicolumn{1}{c}{ day 5} & day 6 & day 7 \\
\hline SL & $2.18 \pm 0.64$ & $2.60 \pm 0.78$ & $1.42 \pm 0.42$ & $1.53 \pm 0.61$ \\
DTL1 & $4.96 \pm 1.46 * *$ & $4.16 \pm 0.15 * *$ & $5.44 \pm 1.63 * * *$ & $4.73 \pm 1.37 * *$ \\
DTL3 & $5.80 \pm 1.75 * *$ & $4.24 \pm 0.05 * *$ & $5.27 \pm 1.54 * * *$ & $4.75 \pm 1.45^{* *}$ \\
\hline
\end{tabular}

STL: single top layer; DTL1: double top layer with second layer applied on day 1; DTL3: double top layer with second layer applied on day 3 . 
Table 2. Biliary excretion indexes (BEI) (\%). Bile canaliculi were visualized with 5-(6)carboxy-2', $7^{\prime}$-dichlorofluorescein (CDF). Areas and fluorescence intensity were determined using ImageJ. Results represent the mean values $( \pm$ SEM) obtained from three pictures in each of two hepatocyte cultures $(n=2)$. Statistical significance between STL and DTL were measured using a one-way ANOVA followed by a Dunnett's test $\left(* \mathrm{p}<0.05 ;{ }^{*} \mathrm{p}<0.01\right.$; $* * * \mathrm{p}<0.001)$

\begin{tabular}{lccccc}
\hline \multicolumn{8}{c}{ BEI (\%) } \\
\hline day 4 & \multicolumn{1}{c}{ day 5} & day 6 & day 7 \\
\hline SL & $47 \pm 6$ & $44 \pm 2$ & $38 \pm 3$ & $21 \pm 4$ \\
DTL1 & $58 \pm 4$ & $60 \pm 2 * *$ & $60 \pm 3 * *$ & $35 \pm 1 *$ \\
DTL3 & $61 \pm 2$ & $59 \pm 1 * *$ & $57 \pm 3 * *$ & $44 \pm 2 * * *$ \\
\hline
\end{tabular}

STL: single top layer; DTL1: double top layer, second layer applied on day 1; DTL3: double top layer, second layer applied on day 3. 
Table 3. Comparison of the Area Under the Curve (AUC) of collagen levels as function of culture time determined in sandwich-cultured rat hepatocytes (SCRH) after a single collagen overlay (STL) or with an double overlay applied on day 1 (DTL1) or day 3 (DTL3). The amount of collagen quantified in the top layer of SCRH in function of culture time, as shown in Figure 5 was used to calculate the AUC values using the trapezoidal rule.

\begin{tabular}{lll}
\hline & $\begin{array}{l}\text { Area Under the Curve } \\
(\text { AUC) } \pm \text { SD of STL1 }\end{array}$ & $\begin{array}{l}\text { Area Under the Curve } \\
(\text { AUC) } \pm \text { SD of DTL }\end{array}$ \\
\hline STL versus DTL1 & $0.71 \pm 0.10$ & $0.90 \pm 0.14$ \\
STL versus DTL3 & $0.41 \pm 0.08$ & $0.61 \pm 0.14$ \\
\hline
\end{tabular}

